\title{
Stress Radiography for the Diagnosis of Medial Glenohumeral Ligament Rupture in Canine Shoulders
}

\author{
Véronique Livet ${ }^{1,2}$ Mathieu Harel ${ }^{3}$ Mathieu Taroni ${ }^{1,2}$ Claude Carozzo ${ }^{1,2}$ Éric Viguier ${ }^{1,2}$ \\ Juliette Sonet ${ }^{3}$ Thibaut Cachon ${ }^{1,2}$ \\ ${ }^{1}$ Department of Small Animal Surgery, Université de Lyon, VetAgro \\ Address for correspondence Véronique Livet, DMV, MSc, VetAgro \\ Sup, Marcy l'Etoile, France \\ 2 Research Unit ICE, UPSP 2016-A104, Université de Lyon, VetAgro \\ Sup, Campus Vétérinaire de Lyon, 1 avenue Bourgelat, 69280 Marcy \\ Sup, Marcy l'Etoile, France \\ ${ }^{3}$ Department of Small Animal Imaging, Université de Lyon, VetAgro \\ Sup, Marcy l'Etoile, France \\ Vet Comp Orthop Traumatol 2019;32:433-439.
}

\begin{abstract}
Objectives This study aimed to describe a stress radiography to detect medial glenohumeral ligament rupture and to investigate the repeatability and reproducibility of this test.

Materials and Methods Six adult Beagle cadavers were used. Both shoulders of each dog were randomly assigned into two groups: a normal shoulder group (NS group) and an affected shoulder group (AS group) where the medial glenohumeral ligament was arthroscopically transected. Goniometry, image analysis and radiography (normal and stress views) were performed before and after arthroscopic procedures. An abduction angle was determined for each stress view and an evaluation of the repeatability and the reproducibility was performed.

Results Abduction angles were significantly larger in the AS group as measured by goniometer $(p<0.001)$, image analysis $(p<0.001)$ and radiographic stress view $(p<0.001)$ than in the NS group. The abduction angle difference varied from $12^{\circ}$ to $29.3^{\circ}$ (median difference of $19.4^{\circ}$ ) between NS and AS groups. There were good intra-observer and very

Keywords

- dogs

- shoulder

- goniometry

- radiography

- arthroscopy good inter-observer agreements. A very good $(r=0.74 ; r=0.72$, respectively) significant $(p<0.001)$ positive correlation between goniometric and radiographic techniques and between image analysis and radiographic techniques was observed.

Clinical Significance Shoulder abduction angles measured with a stress radiographspecific view provide objective data that may contribute to diagnosis of medial shoulder instability in dogs.
\end{abstract}

\section{Introduction}

Shoulder instability is one of the most common causes of shoulder lameness. ${ }^{1,2}$ Medial shoulder subluxation appears to account for approximately $80 \%$ of all shoulder joint instability. ${ }^{3,4}$ The medial glenohumeral ligament is shaped like a Y with both cranial and caudal arms. The medial glenohumeral ligament plays a major role in shoulder stability. One study showed that transection of the medial glenohumeral ligament led to complete medial luxation of the joint in canine cadavers. ${ }^{5}$ Damage to the medial glenohumeral ligament has been reported in $66 \%$ of unstable shoulders. ${ }^{1,6,7}$ received

May 1, 2018

accepted after revision

May 3, 2019 (c) 2019 Georg Thieme Verlag KG Stuttgart · New York
DOI https://doi.org/ $10.1055 / \mathrm{s}-0039-1692469$. ISSN 0932-0814. 
However, diagnostic characterization of shoulder instability in dogs is challenging. Physical and orthopaedic examinations, including manipulation of the glenohumeral joint and measurement of the abduction angle, are considered to be essential. ${ }^{2}$ While Cook and colleagues showed that the abduction angle test appeared reliable for the diagnosis of medial shoulder instability, other authors showed that this test could lead to some false positives and negatives. ${ }^{6,8}$ Thus, it is necessary to use other diagnostic elements to confirm the instability. Additionally, conventional diagnostic imaging can help establish the diagnosis but radiographic examinations is often normal with only secondary degenerative lesions. ${ }^{1}$ Puglisi and colleagues attempted to perform a stress radiographic technique on the scapulohumeral joint and found a significant difference in the mediolateral joint-space measurement between pre- and postoperative stress radiographs. ${ }^{9}$ Thus, arthroscopy, magnetic resonance imaging, magnetic resonance arthrography and computed tomographic arthrography are often required to see shoulder soft tissue structures and diagnose shoulder injuries, but these are less commonly available and are more expensive diagnostic tools. . $^{810-16}$

The objective of our study was to describe a new technique for stress radiography allowing an accurate diagnosis of medial glenohumeral ligament rupture and medial shoulder instability. Moreover, our purpose was to investigate the repeatability (intra-observer agreement) and reproducibility (inter-observer agreement) of this new radiographic technique.

\section{Materials and Methods}

\section{Dogs}

Six Beagle dogs were euthanatized for reasons unrelated to the study. None of the dogs had a history of shoulder disease, and no abnormalities were detected during orthopaedic and radiographic examinations. Body weight was documented. Dog cadavers were allowed to thaw for 24 hours at room temperature before all testing.

\section{Study protocol}

Both shoulders of each dog were randomly assigned into two groups: a normal shoulder group (NS group) and an affected shoulder group (AS group). Shoulder arthroscopies were performed on all shoulders. Each dog was positioned in lateral recumbency with the desired shoulder facing up (away from the table). A $2.7-\mathrm{mm} 30^{\circ}$ oblique arthroscope (Karl Storz; Mittelstrasse, Germany) with a digital camera for video capture was introduced via a standard caudolateral portal. ${ }^{17}$ A craniolateral instrument portal was then established. The intact structure of the medial glenohumeral ligament and the joint capsule was confirmed by probing (-Fig. 1A). For the AS group, the medial glenohumeral ligament (both cranial and caudal arms) was transected transversely on its mid-substance with arthroscopic scissors (-Fig. 1B and 1C). All procedures were performed by one investigator (TC, board-certified surgeon). Goniometry, image analysis and radiographic analysis were performed on each shoulder.

\section{Goniometry and image analysis}

For each shoulder, measurements of the abduction angle were obtained before and after arthroscopy by the use of a universal plastic goniometer and with a digital image taken from the cranial aspect, as described previously. ${ }^{6}$ Both techniques were performed in triplicate by one investigator (VL, resident surgeon), who was completely blinded regarding the allocation of each shoulder.

\section{Radiography}

Initially, two standard radiographic projections (mediolateral and caudocranial) were obtained for each shoulder. An additional stress view was performed: dogs were positioned in dorsal recumbency with the limb to be imaged pulled cranially and parallel to the table and placed in a radiographic positioning device that provided restraint. The device was placed to block the whole body of the animal until the scapula just behind the acromion. The goal was to maintain the scapula against the body and to avoid abduction of this shoulder. One operator performed shoulder abduction with the scapula blocked against the thorax by the radiographic positioning device (-Fig. 2). The shoulder abduction was realized with maximal force without reaching an external elbow rotation. During the abduction, the limb was also
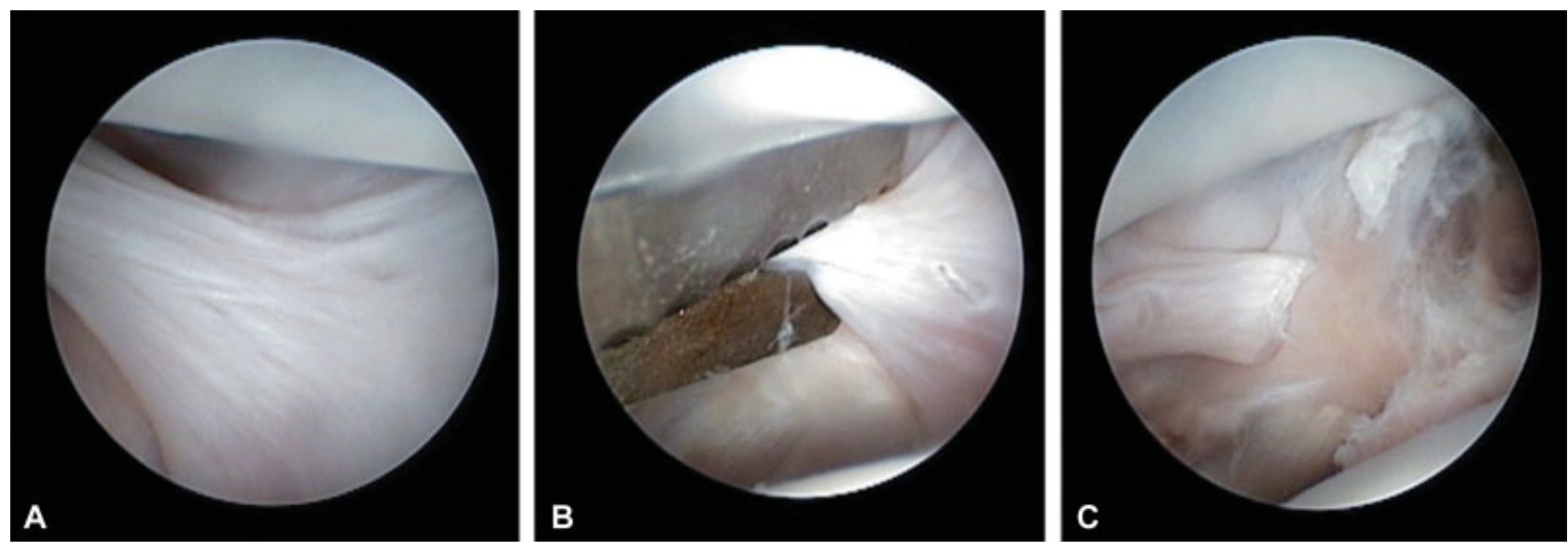

Fig. 1 Visualization of the medial glenohumeral ligament with its ' $\gamma$ ' shape during arthroscopy (A). Transection of the medial glenohumeral ligament with arthroscopic scissors (B). Visualization of the transected medial glenohumeral ligament (C). 

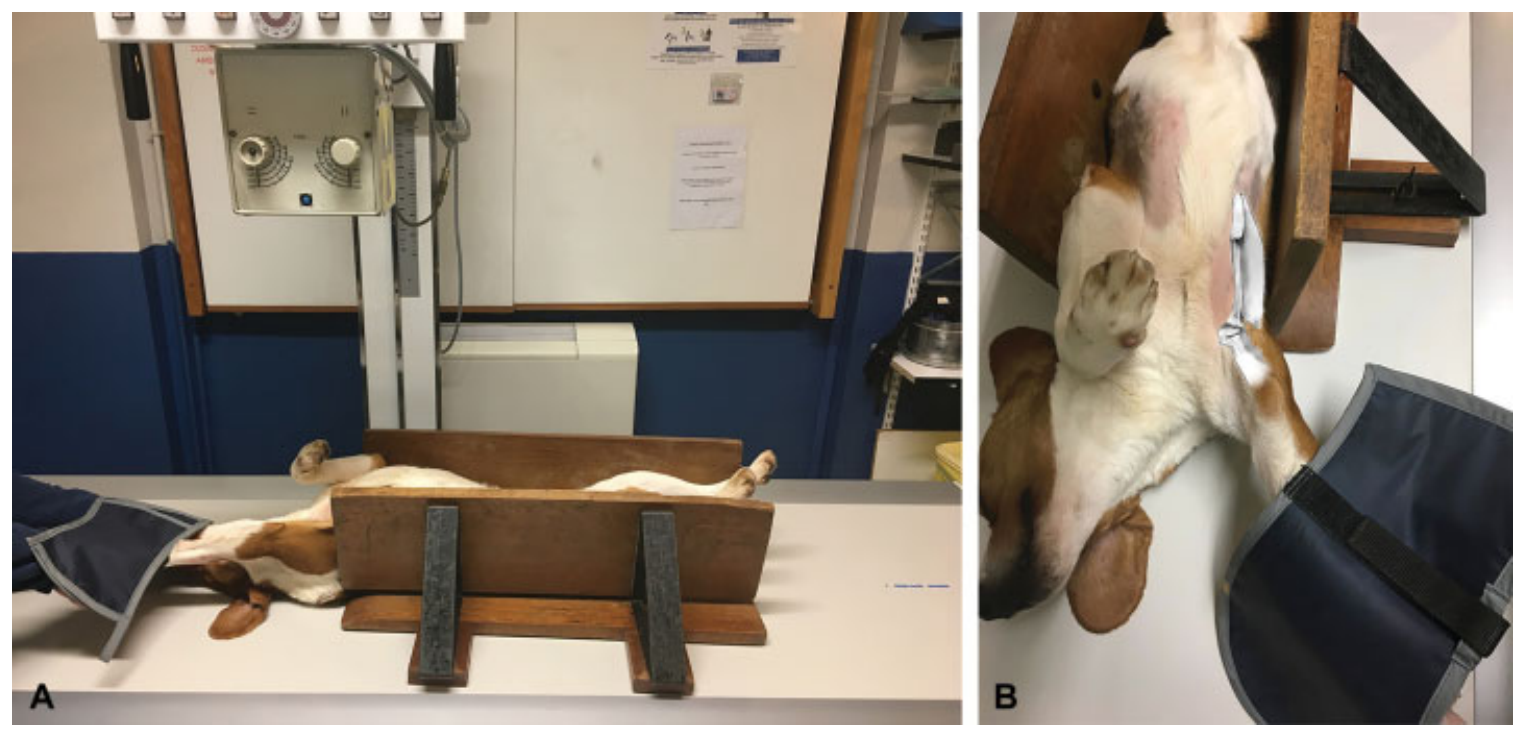

Fig. 2 Realization of a new stress-view radiograph: dogs were positioned in dorsal recumbency and placed in a radiographic positioning device that provided patient restraint. The device was placed to block the whole animal body to the scapula (end of the thorax) (A). One operator performed shoulder abduction with the scapula blocked against the thorax via the radiographic positioning device (B).

pulled cranially and the X-ray beam was positioned perpendicular to the scapula to avoid distraction. The three radiographic views were taken for all shoulders before and after arthroscopy. To test the repeatability of the newly described stress view, three stress views were performed after arthroscopy with an interval of 4 weeks between each view (dog cadavers were thawed and re-frozen each time). All radiographs were performed by two investigators (VL, resident surgeon; $\mathrm{MH}$, resident radiologist) who were blinded regarding the allocation of each shoulder.

\section{Radiographic measurements}

The distal scapular anatomic axis was determined on the stress-view radiograph: the midpoint between the medial and lateral cortices of the scapular body at the point $1 / 3$ down the length of the scapula and the centre of the medial and lateral cortices at the scapular neck were identified. The distal scapular anatomic axis was represented by the line connecting these two points. The proximal humeral anatomic axis was determined on the same radiograph: the midpoint between the medial and lateral cortices of the humerus at the point halfway down the length of the humerus and the centre of the medial and lateral cortices at a point $1 / 3$ down the length of the humerus were identified. The proximal humeral anatomic axis was represented by the line connecting these two points. The abduction angle was determined by the angle between these two lines (- Fig. 3). Measurements were performed in triplicate for each stress-view radiograph by one investigator (VL, resident surgeon) before arthroscopy, and by three different investigators (TC, board-certified surgeon; VL, resident surgeon; $\mathrm{MH}$, resident radiologist) after arthroscopy (three stress views per shoulder). All stress-view radiographs were shuffled before reading, and a minimum interval of 1 week between each reading was respected. A standard imaging

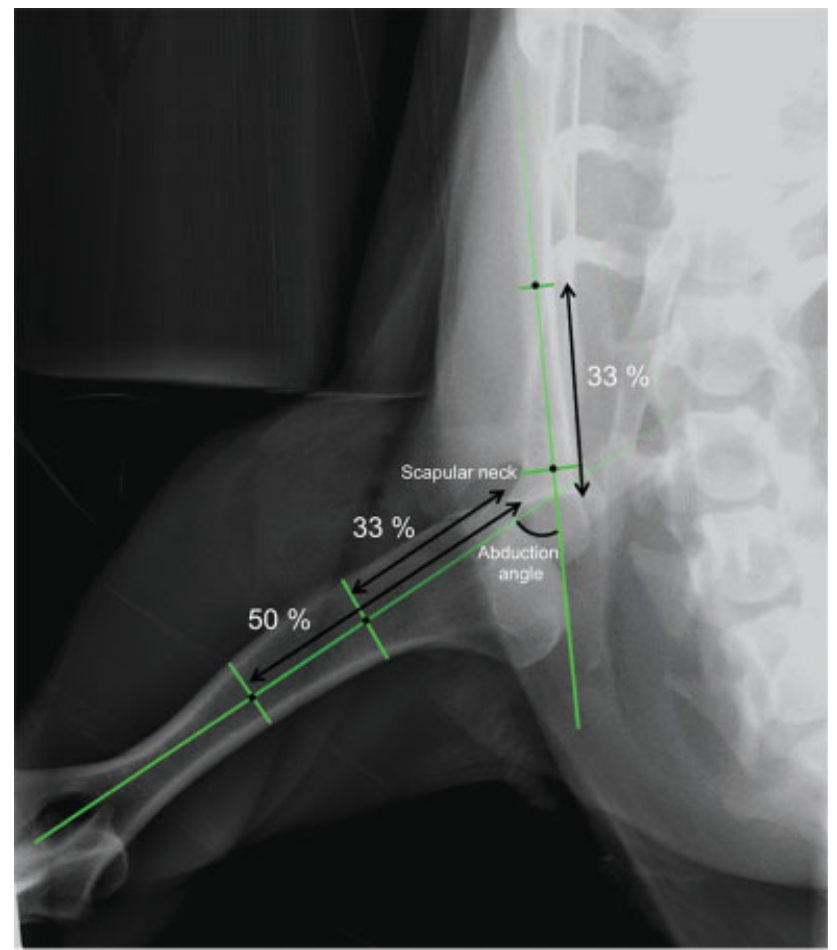

Fig. 3 Abduction angle measurement on a stress-view caudocranial radiograph. The distal scapular anatomical axis was determined: the midpoint between the medial and lateral cortices of the scapular body at the point $1 / 3$ down the length of the scapula and the centre of the medial and lateral cortices at the scapular neck were identified. The distal anatomical axis was represented by the line relative to these two points. The proximal humeral anatomical axis was determined: the midpoint between the medial and lateral cortices of the humerus at the point halfway down the length of the humerus and the centre of the medial and lateral cortices at a point $1 / 3$ down the length of the humerus were identified. The proximal anatomical axis was represented by the line relative to these two points. The abduction angle was determined by the angle between these two lines. 
software was used for measurements (Osirix; Pixmeo, Geneva, Switzerland).

\section{Recorded data and statistical analysis}

The normality of numerical data distribution was tested by visual approach and D'Agostino \& Pearson omnibus normality test. For clarity, all numerical data were expressed as median and range. Comparisons were performed with the Mann-Whitney $U$ test for non-normally distributed data and with the Student's $t$-test for normally distributed data. Qualitative data were compared by the Fisher's exact test. Spearman's correlation testing was used to determine if significant correlations existed between the three techniques used for determining abduction angles in this study. A $p$-value of less than 0.05 was considered significant. All analyses were performed using commercial software (Prism 6.0e; GraphPad Software Inc, La Jolla, California, United States).

To evaluate intra-observer and inter-observer agreement, statistical analyses were performed with the software $R$ 3.2.3. The intra- and inter-observer agreements of postoperative angles were evaluated with the overall concordance correlation coefficient (CCC) and the unified agreement approach, respectively (Packages epiR, Agreement). ${ }^{18-21}$ The CCC varies between -1 and 1 ; a CCC of 1 indicates perfect repeatability or perfect agreement. ${ }^{22}$

\section{Results}

\section{Study cases}

Four of the Beagle dogs were female and two were male. The median age was 16 months (range: 13-20 months) and the median weight was $12.2 \mathrm{~kg}$ (range: 10.3-13.7 kg). No abnormality was seen in any shoulder joint on initial radiographs and during the arthroscopic procedure.

\section{Goniometry and image analysis}

Median abduction angles and ranges measured by goniometry and image analysis before and after arthroscopy in both groups are shown in - Table 1. No significant differences were noted in the measured abduction angles before arthroscopy between the AS and NS groups for goniometry $(p=0.06)$ or image analysis $(p=0.92)$. No significant differences were found in the measured abduction angles before and after arthroscopy in the NS group for goniometry $(p=0.34)$ or image analysis $(p=0.86)$. Abduction angles were significantly larger in the AS group after the arthro- scopic procedure by both goniometry $(p<0.001)$ and image analysis $(p<0.001)$. Abduction angles were significantly larger in the AS group compared with the NS group after the arthroscopic procedure by goniometry $(p<0.001)$ and image analysis ( $p<0.001)$ ( - Fig. 4). Moreover, when comparing NS and AS for each dog, abduction angles were significantly larger for the AS than the NS after the arthroscopy procedure by goniometry $(p<0.001)$ and image analysis $(p<0.001)$. The abduction angle varied from $6^{\circ}$ to $33^{\circ}$ (median difference of $16^{\circ}$ ) with goniometry and from 8 to $32^{\circ}$ (median difference of $17^{\circ}$ ) with image analysis between the NS and AS after arthroscopy. Very good $(r=0.79)$ significant $(p<0.001)$ positive correlation between the goniometric and image analysis techniques was observed.

\section{Radiography}

Mediolateral and caudocranial radiographic views were unremarkable. Median abduction angles and ranges measured on radiographs before and after arthroscopy in both groups are provided in - Table 1. No significant differences were noted in the measured abduction angles before arthroscopy between the AS and NS groups for radiographic measurements $(p=0.06)$. No significant differences were found in the measured abduction angles before and after arthroscopy in the NS group on radiographic exam $(p=0.94)$. Abduction angles were significantly larger in the AS group after the arthroscopic procedure with radiographic stress views $(p<0.001)$. Abduction angles were significantly larger in the AS group compared with the NS group after the arthroscopic procedure with radiographic measurements $(p<0.001)$ ( - Fig. 4). Moreover, when comparing NS and AS for each dog, abduction angles were significantly larger for the AS than the NS after the arthroscopy procedure on radiographic examination $(p<0.001)$. The median difference in abduction angle between both sides after arthroscopy was $19.4^{\circ}$ (range: $12^{\circ}-29.3^{\circ}$ ). Very good $(r=0.74)$ significant $(p<0.001)$ positive correlation between goniometric and radiographic techniques was observed. Very good significant positive correlation $(r=0.72 ; p<0.001)$ between image analysis and radiographic techniques was also found.

Intra- and inter-observer agreement-For preoperative shoulder abduction angles on radiographs, there was moderate-to-good intra-observer agreement, with good precision and excellent accuracy $(C C C=0.80$; precision $=0.83$; accuracy $=0.96$ ). For postoperative shoulder abduction angles, there were good intra-observer agreement (CCC

Table 1 Median (range) abduction angles measured by goniometry, image analysis and radiography for normal shoulders and affected shoulders with medial glenohumeral ligament rupture

\begin{tabular}{|l|l|l|l|l|}
\hline & \multicolumn{2}{|l|}{ NS group } & \multicolumn{2}{l|}{ AS group } \\
\hline & Before arthroscopy & After arthroscopy & Before arthroscopy & After arthroscopy \\
\hline Goniometry (degree) & $20.0(14.0-28.0)$ & $21.0(18.0-28.0)$ & $23.5(18.0-30.0)$ & $42.0(30.0-52.0)$ \\
\hline Image analysis (degree) & $25.0(14.0-32.0)$ & $24.0(18.0-30.0)$ & $24.0(18.0-28.0)$ & $46.0(30.0-52.0)$ \\
\hline Radiography (degree) & $36.6(31.5-42.0)$ & $36.7(30.2-43.4)$ & $37.5(31.9-44.7)$ & $53.0(42.6-60.7)$ \\
\hline
\end{tabular}

Abbreviations: AS, affected shoulder; NS, normal shoulder. 


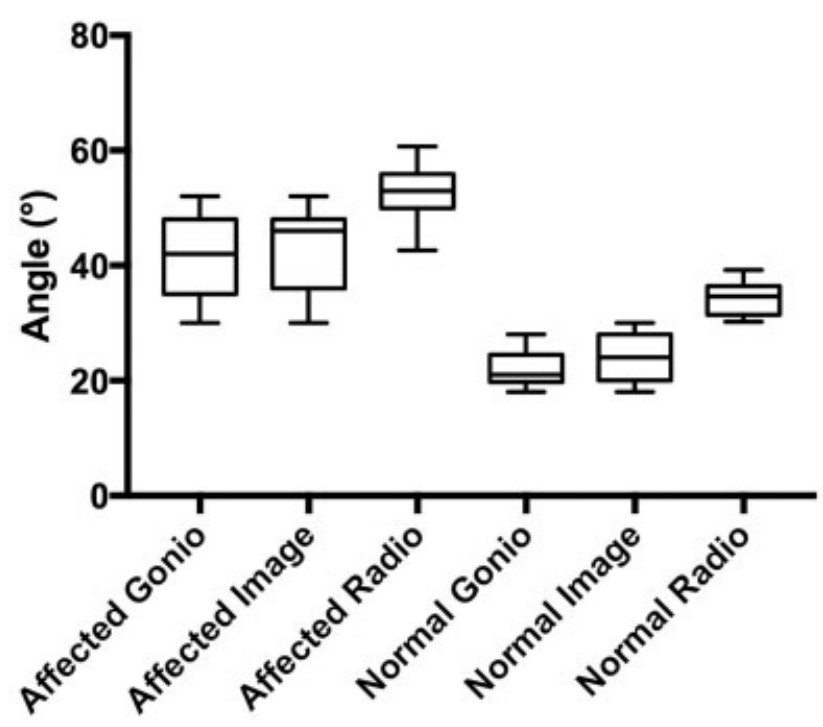

Fig. 4 Box plot showing shoulder abduction angles for the affected shoulder group and normal shoulder group after arthroscopy using goniometric (gonio), image analysis (image) and radiographic (radio) techniques. The line transecting the box is the median, and the boundaries of the box show the 25 th and 75 th percentiles.

$=0.95$; precision $=0.95$; accuracy $=1)$ and very good interobserver agreement $(\mathrm{CCC}=0.98$; precision $=0.98$; accuracy $=1)$. Although the precision of the measurements was not perfect, the accuracy was nearly perfect.

\section{Discussion}

This cadaveric study describes a new stress radiographic view that allowed consistent diagnosis of medial glenohumeral ligament rupture, with AS having an increased scapulohumeral abduction angle on radiographic measurements compared with shoulders of the normal control group. Median abduction angle measured on radiographs was $36.7^{\circ}$ (range: $30.2^{\circ}-43.4^{\circ}$ ) after arthroscopy for NS and $53^{\circ}$ (range: $42.6^{\circ}-60.7^{\circ}$ ) for AS. The difference in abduction angle for each dog between the AS and NS varied from 12 to $29.3^{\circ}$ (median difference of $19.4^{\circ}$ ) after arthroscopy for radiographic measurements. Abduction angles were significantly larger for AS compared with NS after the arthroscopy procedure on radiographs $(p<0.001)$.

According to Cook and colleagues, a hyperabduction angle of the shoulder $>50^{\circ}$ measured by goniometry and image analysis is considered positive for medial shoulder instability. ${ }^{6}$ In our study, the median abduction angle measured by goniometry was $42^{\circ}$ (range: $3^{\circ} 0-52^{\circ}$ ) for AS. Even if these results were significantly larger than before medial glenohumeral ligament transection, the hyperabduction test was positive for only one dog with this method. In the same study, the comparison of AS and NS appeared to be more relevant than a single absolute value of the abduction angle. Cook and colleagues found a relatively large difference in abduction angles $\left(>20^{\circ}\right)$ between AS and NS during goniometry and imaging analysis. ${ }^{6}$ In our study, a median difference of $16^{\circ}$ (range: $6^{\circ}-33^{\circ}$ ) with goniometry was observed. Even if the abduction angle difference between the NS and AS appears smaller in our cadaveric study for goniometry and image analysis, a significant difference was nevertheless found. This difference between our result and Cook and colleagues could be due to the use of cadavers, as well as the fact that only the medial glenohumeral ligament was transected in our study in contrast to Cook and colleagues' study, wherein muscles such as the subscapularis were also severed. Moreover, Cook's study included 15 different breeds (mean body weight of $36.3 \mathrm{~kg}$ ), whereas our study used a homogeneous population with only Beagles (mean body weight of $12.0 \mathrm{~kg}$ ).

Even if Cook and colleagues showed that shoulder abduction angles measured under sedation provide objective data for diagnosis of shoulder instability in dogs, ${ }^{6}$ Devitt and colleagues found that a negative hyperabduction test was 7.14 times more likely in a dog without medial compartment changes than in a dog with a positive hyperabduction test. ${ }^{8}$ These investigators also showed that the abduction angle test had good sensitivity but low specificity. Thus, while an abduction angle $>50^{\circ}$ may be a consistent feature of medial shoulder instability, it may also be a consistent feature of any disorder of the thoracic limb that results in significant loss in muscle strength or muscle mass. The results of Cook and colleagues' work are valuable in establishing reference ranges for abduction angles of the shoulder, yet notable limitations to that study exist. For example, the control group was not subjected to the reference standard test of arthroscopic evaluation. Failing to subject the control group to the same reference standard does not allow for a valid comparison of a diagnostic test. A radiographic evaluation is therefore relevant to support a positive hyperabduction test. Moreover, radiography remains the most common imaging modality used for investigating thoracic lameness. Radiography is readily accessible, inexpensive and can be performed after sedation with minimal morbidity.

Based on our results, comparison between radiographic abduction angles of both shoulders could be an interesting value to diagnose medial shoulder instability. However, in clinical setting, when a bilateral shoulder affection is suspected, the test reported here could not be used in that way. Thus, normal radiographic abduction angles need to be determined in a larger population and with different breeds of dog to be used as a cut-off for the diagnosis of medial shoulder instability.

In our study, the median abduction angle measured with the new stress radiographic view was $53^{\circ}$ (range: $42.6^{\circ}-$ $60.7^{\circ}$ ) after medial glenohumeral ligament rupture in the AS. Abduction angles were significantly larger after medial glenohumeral ligament rupture $(p<0.001)$. Compared with Cook and colleagues' goniometry results (53.7 \pm 4.7 ), abduction angles measured by radiographs were closer than abduction angles measured by goniometry in our study. ${ }^{6}$ Very good significant correlation was found between goniometry and imaging analysis measurements and radiographic measurements, allowing this new diagnostic tool to be considered reliable. Radiographic abduction angle results were within the reference range compared with other shoulder abduction studies. ${ }^{6}$ Advantages of radiographic 
measurements include a better repeatability of the radiographic measurements compared with the use of a universal plastic goniometer. In fact, the radiographic measurements performed in the present study used specific landmarks, such as specific bone reliefs and bone lengths, whereas goniometry uses external landmarks that are less reliable and more difficult to reproduce. Disadvantages include difficult and critical positioning to avoid rotation of the limb during abduction.

Puglisi and colleagues attempted to perform a stress radiographic technique on the scapulohumeral joint of 20 small dogs. ${ }^{9}$ Stress radiographs (mediolateral and caudocranial views) were taken using approximately $11 \mathrm{~kg}$ of force before and after bilateral medial instability surgically created in six dogs. No differences were noted in four measurements of the scapulohumeral joint between survey radiographs and preoperative stress radiographs. A significant difference was found in the mediolateral joint-space measurement between pre- and postoperative stress radiographs. On the caudocranial view, no significant difference was found between preand postoperative radiographs. In our study, we used a caudocranial stress radiographic view that created an abduction of the scapulohumeral joint, which was different compared with Puglisi and colleagues. Our results confirmed that different stress radiographic views of the scapulohumeral joint allowed a precise and simple diagnosis of medial shoulder instability. However, we did not use a standardized force like Puglisi and colleagues, because the abduction was performed by an operator, and because no device exists to realize such stress views.

Intra-observer and inter-observer agreements were very good in our study. The three investigators had different experience levels with shoulder pathologies and shoulder radiographs (one board-certified surgeon, one resident surgeon and one resident radiologist), but no differences occurred between measurements.

In our study, we did not measure the force applied during the radiographic abduction test which could have been interesting to improve the repeatability of the test. However, without a force measurement, it can be included in every day practice. The maximal force to abduct the shoulder without an elbow rotation was chosen as in the abduction angle test described by Cook and colleagues. ${ }^{6}$ Finally, the force necessary to induce a maximal shoulder abduction is not known and could be breed specific.

Our study is limited by its use of cadavers and small sample size. This study used only Beagle dogs, and thus limited the conclusions to only this small-breed dog. One of the most important limitations is our model, based on an acute lesion for all the medial glenohumeral ligaments that may not fully represent the great variety of lesions that can be found in living dogs. The anatomical landmarks that we chose were not described before, and the accuracy of these measurements remains to be determined. We used a similar radiographic method previously developed for the canine radius and femur and we hypothesized that it could be applied for the humerus and the scapula to determine the anatomical axis of those bones. $^{23,24}$ Investigators were all specialists or residents, and measurements by non-board-certified veterinarians could have been performed to confirm the reproducibility of this method. In our study, the repeatability of the positioning was good as all the measurements performed on three different views were similar. Nevertheless, radiographs were realized by the same two investigators. Repeatability of the positioning should be assessed with other operators. The new radiographic method also requires the presence of an operator performing abduction of the shoulder during the taking of the radiograph. Therefore, this implies ionizing radiation exposure that is contrary to radiation protection and that is not compatible with law in several countries. One method to apply the same constraint without an operator would be interesting to develop to be used without concern for associated radiation. Finally, this technique requires a specific device to position the animal and its forelimb and anaesthesia is required to realize the stress view. However, this device is quite simple and initially used in our practice to perform stress radiograph of the hips by blocking the whole body. Therefore, any device that can maintain an adequate lateral pressure on the dog's body and scapula can be used.

Because medial instability is reported to comprise the largest percentage of shoulder instability cases in dogs, we sought to develop an objective diagnostic technique that would specifically address this type of pathology through a cadaver pilot study. ${ }^{1}$ We conclude that shoulder abduction angles measured with a stress radiographic-specific view provide objective data that may contribute to the diagnosis of medial shoulder instability in dogs. Shoulder abduction angles are significantly larger in AS with the medial glenohumeral ligament transected than in NS in beagle dogs. Future clinical prospective studies are needed to determine how this additional diagnostic information should influence the diagnostic approach of shoulder lameness.

\section{Author Contribution}

Véronique Livet, Thibaut Cachon and Juliette Sonet contributed to conception of study, study design, acquisition of data and data analysis and interpretation. Mathieu Harel contributed to study design, acquisition of data and data analysis and interpretation. Mathieu Taroni contributed to data analysis and interpretation. Claude Carozzo contributed to conception of study and data analysis and interpretation. Éric Viguier contributed to conception of study. All authors drafted, revised and approved the submitted manuscript.

\section{Funding}

The authors received no financial support for the research, authorship and/or publication of this article.

\section{Conflict of Interest}

The authors have no conflict of interests with regard to this manuscript.

\section{Acknowledgments}

The authors thank Dr. Anthony Barthélemy and Dr. Sylvain Larrat for assistance with statistical analyses. 


\section{References}

1 Bardet JF. Diagnosis of shoulder instability in dogs and cats: a retrospective study. J Am Anim Hosp Assoc 1998;34(01):42-54

2 Cogar SM, Cook CR, Curry SL, Grandis A, Cook JL. Prospective evaluation of techniques for differentiating shoulder pathology as a source of forelimb lameness in medium and large breed dogs. Vet Surg 2008;37(02):132-141

3 Bardet JF. Shoulder diseases in dogs. Vet Med 2002;97:909-918

4 Rochat MC. The shoulder. In: Johnston SA, Tobias KM, eds. Veterinary Surgery: Small Animal. Vol 1. 2nd ed. St Louis: Elsevier; 2017:800-820

5 Sidaway BK, McLaughlin RM, Elder SH, Boyle CR, Silverman EB. Role of the tendons of the biceps brachii and infraspinatus muscles and the medial glenohumeral ligament in the maintenance of passive shoulder joint stability in dogs. Am J Vet Res 2004;65(09):1216-1222

6 Cook JL, Renfro DC, Tomlinson JL, Sorensen JE. Measurement of angles of abduction for diagnosis of shoulder instability in dogs using goniometry and digital image analysis. Vet Surg 2005;34 (05):463-468

7 Cook JL, Tomlinson JL, Fox DB, Kenter K, Cook CR. Treatment of dogs diagnosed with medial shoulder instability using radiofrequency-induced thermal capsulorrhaphy. Vet Surg 2005;34 (05):469-475

8 Devitt CM, Neely MR, Vanvechten BJ. Relationship of physical examination test of shoulder instability to arthroscopic findings in dogs. Vet Surg 2007;36(07):661-668

9 Puglisi TA, Tanger CH, Green RW, et al. Stress radiography of the canine humeral joint. J Am Anim Hosp Assoc 1988;24:235-240

10 Schaefer SL, Baumel CA, Gerbig JR, Forrest LJ. Direct magnetic resonance arthrography of the canine shoulder. Vet Radiol Ultrasound 2010;51(04):391-396

11 Schaefer SL, Forrest LJ. Magnetic resonance imaging of the canine shoulder: an anatomic study. Vet Surg 2006;35(08):721-728

12 Reis Silva H, Uosyte R, Clements DN, Bergkvist GT, Schwarz T. Computed tomography and positive contrast computed tomographic arthrography of the canine shoulder: normal anatomy and effects of limb position on visibility of soft tissue structures. Vet Radiol Ultrasound 2013;54(05):470-477
13 De Rycke L, van Bree H, Van Caelenberg A, Polis I, Duchateau L, Gielen I. Epinephrine-enhanced computed tomographic arthrography of the canine shoulder. Res Vet Sci 2015;102:15-21

14 De Rycke LM, Gielen IM, Dingemanse W, Kromhout K, van Bree H. Computed tomographic and low-field magnetic resonance arthrography: a comparison of techniques for observing intraarticular structures of the normal canine shoulder. Vet Surg 2015; 44(06):704-712

15 Agnello KA, Puchalski SM, Wisner ER, Schulz KS, Kapatkin AS. Effect of positioning, scan plane, and arthrography on visibility of periarticular canine shoulder soft tissue structures on magnetic resonance images. Vet Radiol Ultrasound 2008;49(06): 529-539

16 Murphy SE, Ballegeer EA, Forrest LJ, Schaefer SL. Magnetic resonance imaging findings in dogs with confirmed shoulder pathology. Vet Surg 2008;37(07):631-638

17 Beale BS, Hulse DA, Schulz KS, et al. Shoulder arthroscopy. In: Beale BS, Hulse DA, Schulz KS, et al, eds. Small Animal Arthroscopy. 1st ed. Philadelphia: Saunders; 2003:23-49

18 Lin L. A note on the concordance correlation coefficient. Biometrics 2000;56:324-325

19 Lin LI. A concordance correlation coefficient to evaluate reproducibility. Biometrics 1989;45(01):255-268

20 Barnhart HX, Haber M, Song J. Overall concordance correlation coefficient for evaluating agreement among multiple observers. Biometrics 2002;58(04):1020-1027

21 Lin L, Hedayat AS, Wu W. A unified approach for assessing agreement for continuous and categorical data. J Biopharm Stat 2007;17(04):629-652

22 Dohoo IR, Martin SW, Stryhn H. Screening and diagnostic tests. In: Dohoo IR, Martin SW, Stryhn H, eds. Veterinary Epidemiologic Research. 2nd ed. Charlottetown: AVC Incorporation; 2003: 92-135

23 Tomlinson J, Fox D, Cook JL, Keller GG. Measurement of femoral angles in four dog breeds. Vet Surg 2007;36(06):593-598

24 Fox DB, Tomlinson JL, Cook JL, Breshears LM. Principles of uniapical and biapical radial deformity correction using dome osteotomies and the center of rotation of angulation methodology in dogs. Vet Surg 2006;35(01):67-77 